\title{
Systematic errors in measuring solar magnetic fields with a FPI spectrometer and MDI
}

\author{
A. Settele ${ }^{1}$, T. A. Carroll ${ }^{1}$, I. Nickelt ${ }^{1}$, and A. A. Norton ${ }^{2}$ \\ 1 Astrophysikalisches Institut Potsdam, Telegrafenberg A27, 14473 Potsdam, Germany \\ 2 W. W. Hansen Experimental Physics Laboratory, Center for Space Science and Astrophysics, \\ Stanford University, CA 94305-4085, USA
}

Received 10 April 2001 / Accepted 28 February 2002

\begin{abstract}
We will show the potential errors of observations using instruments, where a line spectrum is obtained not instantly but by scanning through the spectral line in time. We give two examples in simulations of a sunspot observation with the measuring procedure of a typical Fabry-Perot interferometer (FPI) spectrometer and the Michelson Doppler Imager (MDI) on board of the Solar and Heliospheric Observatory (SOHO). The effect described is inherent for observations with a low intensity input signal, where the accumulation time for one image and therefore the whole process of measuring a spectrum is relatively high compared to the timescale of the underlying physical processes. We show that our simulated data produce an error in magnetic field strength and - in a time series - result in an apparent oscillation of the magnetic field strength, while the input contained only velocity oscillations. As the FPI spectrometer is being used more and more widely, one has to be aware of the disadvantages and potential errors when interpreting its data. We encourage every observer to do the simple simulation as described for every specific setup used.
\end{abstract}

Key words. Sun: oscillations, magnetic fields, sunspots - instrumentation: interferometers - techniques: spectroscopic, polarimetric

\section{Introduction}

There are two popular methods to record a spectral line. Conventionally a slit and a grating is used. In this case one obtains the whole line profile simultaneously but only in one spatial dimension. To obtain a two dimensional spatial image the object's picture must be moved over the slit. This disadvantage can be avoided by taking images with a large field-of-view using a system of filters and optical components to effectively sample the spectral line for a few fixed wavelength positions (e.g. the Michelson Doppler Imager, MDI on board of SOHO, Scherrer et al. 1995) or by stepping through the line to achieve a higher spectral resolution (Fabry-Perot interferometer spectrometer, FPI). In this second case we can observe a large spatial field in exchange for the disadvantage of not measuring the spectral line instantaneously. Unfortunately, during the time of measurement the physical process which is to be extracted still develops, e.g. waves running through the solar atmosphere, and the shape of the observed line is deformed or shifted in wavelength.

Send offprint requests to: A. Settele, e-mail: asettele@aip.de
As our ambition is to measure smaller and smaller effects, for example magnetic field oscillations in sunspots, one has to be more and more careful not to interpret artifacts of the measuring process as physical effects. We give one simple example on how a normal velocity oscillation can apparently produce a magnetic field oscillation of a strength in the order of magnitude of those being published in a number of recent observations.

In the following we will first consider the FPI spectrometer with its help we will discuss the origin of the effect, and afterwards we will show results of the simulations of this cross-talk with MDI.

\section{FPI-simulation}

\subsection{Simulation}

We took an artificial Stokes $V$ profile of FeI $617.3 \mathrm{~nm}$ $\left(g_{\text {eff }}=2.5\right)$ calculated by the Stokes profile code DIAMAG (Grossman-Doerth 1994) with an umbral model atmosphere (Kollatschny et al. 1980) pervaded by a $2000 \mathrm{G}$ magnetic field. This profile was shifted in wavelength by a sine wave with different amplitudes and periods. We then simulated the measurement procedure of a FPI spectrometer for a sunspot very similar to the real data acquisition of 


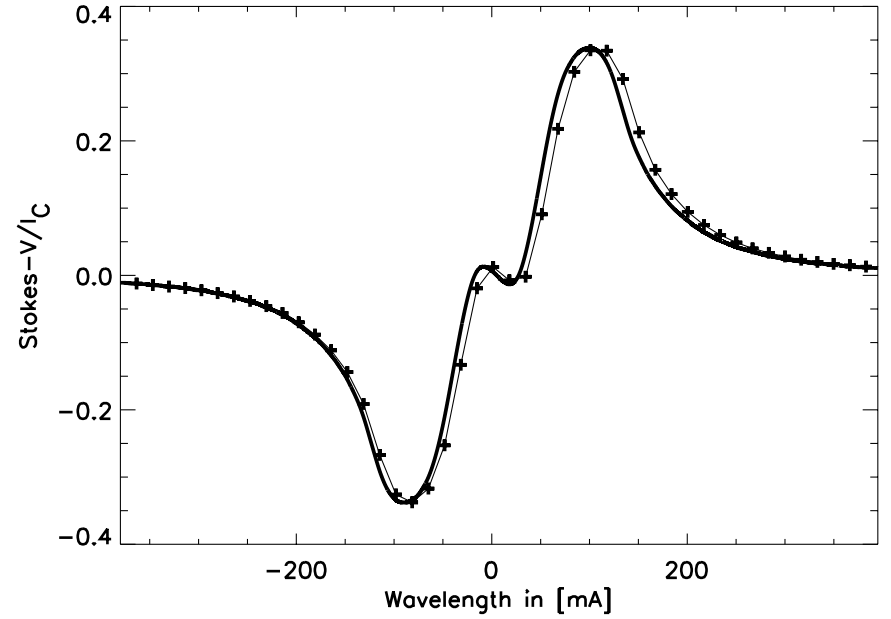

Fig. 1. The original Stokes $V$ profile (thick solid) is stretched by the measuring method. This example is calculated for a 3 min oscillation with a high input velocity amplitude of $1000 \mathrm{~m} \mathrm{~s}^{-1}$, a FPI step size of $16.59 \mathrm{~m} \AA$ and a prescribed field strength of $2000 \mathrm{G}$ to show the effect, in general the deformation is much smaller but nevertheless leads to errors. The profile is normalized to the continuum intensity $\left(I_{\mathrm{C}}\right)$.

the FPI of the Universitätssternwarte Göttingen (Bendlin et al. 1992; Bendlin \& Volmer 1995; Horn et al. 1996), installed at the Vacuum Tower Telescope on Tenerife. This experimental setup to observe a sunspot has the disadvantage that a relatively long exposure time is necessary. We simulated the typical parameters: 50 positions along the wavelength coordinate are subsequently taken with a fixed step width scanning from lower to higher wavelengths. The whole duration of the scan was set to $22.5 \mathrm{~s}$ while the pause between the scans was $3 \mathrm{~s}$, for saving the data. During this measuring process the profile is changing due to the change in velocity. With this setup we produced time series.

In general the FPI does not measure a real Stokes $V$ profile, but two $(I+V)$ and $(I-V)$ profiles simultaneously. In our simulation we used only the Stokes $V$ profile for simplicity, having no effect on the results of the simulation.

\subsection{Simple data analysis}

We used two methods to derive the magnetic field: The first method (hereafter method A) is a simple form of analysis which is also used by some observers: we derived the magnetic fields by the separation of the two Stokes $V$ lobes and the strong field approach.

$$
B=0.5 \cdot \Delta \lambda / 4.67 \times 10^{-16} / g_{\mathrm{eff}} / \lambda^{2},
$$

where $B$ gives the magnetic field strength in $\mathrm{G}, \Delta \lambda$ is the separation of the Stokes $V$ lobes in $\mathrm{m} \AA, g_{\text {eff }}$ is the Landéfactor and $\lambda$ is the line center in $m \AA$. We derive a magnetic field strength of $2161 \mathrm{G}$ of the undisturbed profile by this method.

The velocity was also derived from the two Stokes $V$ lobes by using the center position between both extrema.

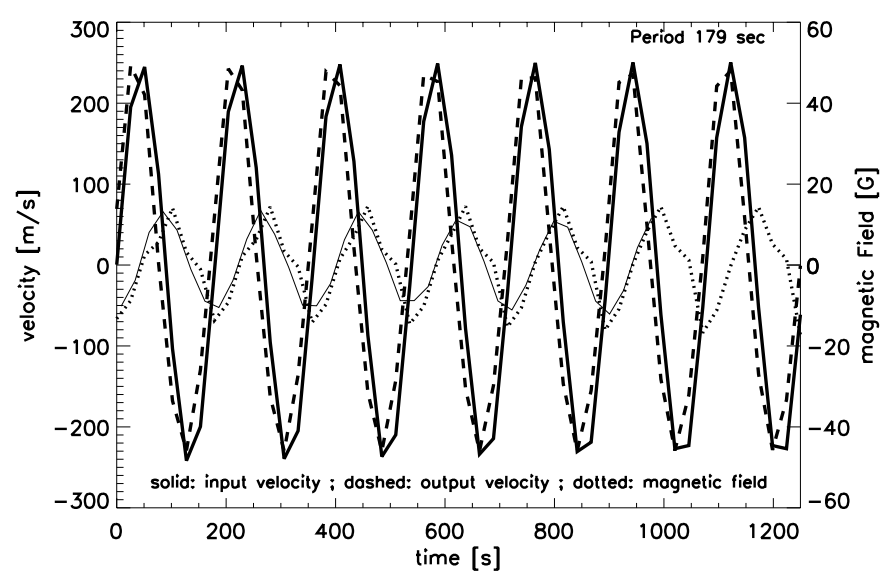

Fig. 2. An example of a 3 min velocity oscillation and the resulting magnetic field oscillation. Input velocity (solid) amplitude: $250 \mathrm{~ms}^{-1}$, FPI step size: $16.59 \mathrm{~m} \AA$. Magnetic field strength: $2000 \mathrm{G}$. The measured velocity is dashed, the measured magnetic field with method (A) is dotted and with method B (inversion) is drawn thin solid.

Note that the fitting routine of the extrema has a large influence on the results as the magnetic field derived by this method is very sensitive on small shifts. We used a forth order polynomial and took 3 points to the left and right of the extrema for fitting equivalent to about $100 \mathrm{~m} \AA$.

To confirm our results we also applied our inversion code (hereafter method B) which is based on response functions. The forward model or synthetic core of the inversion code is a modified version of the DIAMAG code, and the non-linear least square procedure is based on the Levenberg-Marquardt algorithm. The required derivatives of the free parameters are provided in terms of response functions. Although the inversion code is capable to retrieve a depth-dependent stratification, we found that the produced asymmetry of the FPI scanning process do not harbour sufficient information to give rise for a significant depth dependency of the field strength or velocity. For a better comparison of the results we therefore assume no variation in depth for the free parameters in our inversion. We applied the inversion algorithms to the same set of Stokes $V$ profiles of the simulated time series and found the same results in terms of artificial magnetic field strength oscillation and phase relation.

\subsection{Results and discussion}

To underline the effect we want to demonstrate, Fig. 1 shows an enhanced calculation. The FPI step size was set to $16.59 \mathrm{~m} \AA$ and the velocity amplitude of a 3 min oscillation was set to $1000 \mathrm{~m} \mathrm{~s}^{-1}$. The scanning method of the FPI stretched the original profile (thick-solid) and brought back the measured profile (thin-solid). The crosses belong to the measured values. In this case the profile was stretched by the increasing velocity amplitude during the scanning process. It could be also the other way round, that the profile is compressed by a decreasing velocity amplitude. 


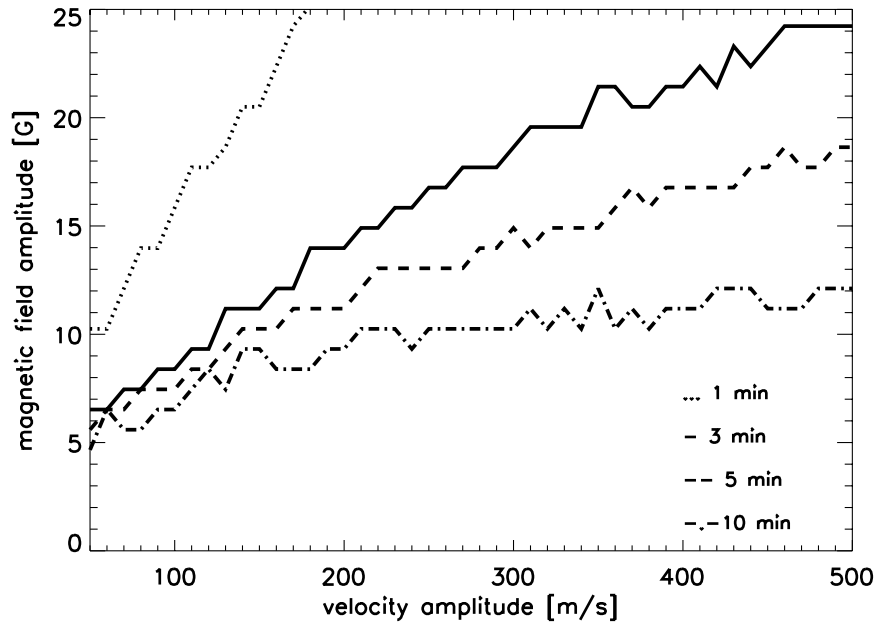

Fig. 3. The magnetic field amplitude is plotted against the velocity of the oscillation for different periods of oscillations calculated with method (A). Note: the amplitude should be zero as there is no oscillating magnetic field. Prescribed magnetic field strength $2000 \mathrm{G}$.

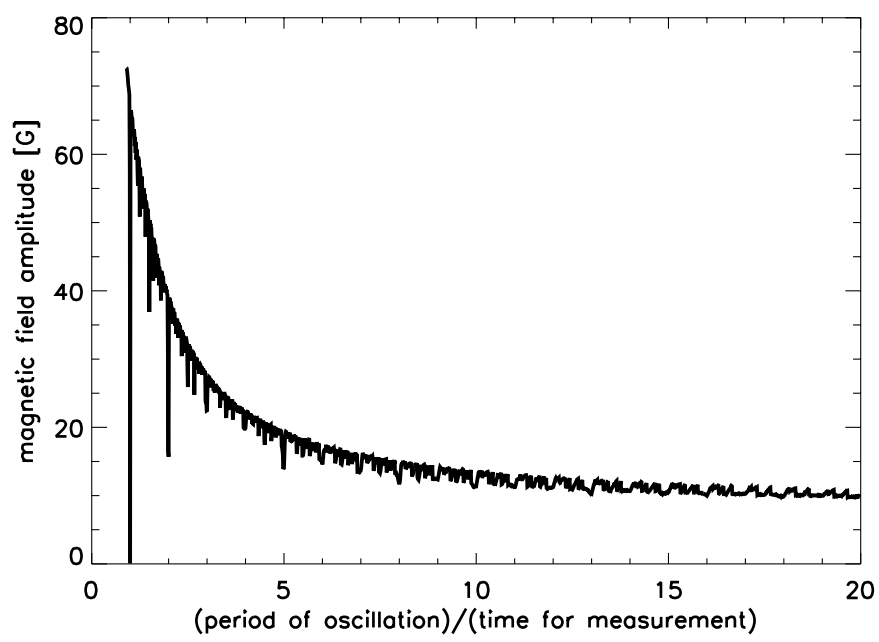

Fig. 4. Erroneous magnetic field in dependence of the period of velocity oscillation. The velocity amplitude was $250 \mathrm{~m} \mathrm{~s}^{-1}$ and the magnetic field $2000 \mathrm{G}$.

Figure 2 shows an example calculation with an input period of $3 \mathrm{~min}$ and a (large) velocity oscillation of $250 \mathrm{~m} \mathrm{~s}^{-1}$ amplitude. The FPI step size was set to $16.59 \mathrm{~m} \AA$. We derive a mean magnetic field of $\approx 2080 \mathrm{G}$ and a peak-to-peak value of $\approx 32 \mathrm{G}$ of the magnetic field oscillation with the method (A). The input velocity plotted (solid) is the velocity at the beginning of the scan process while the output velocity (dashed) is derived from the process simulation. The resulting velocity is generally correct, it is only slightly reduced at $\approx 240 \mathrm{~m} \mathrm{~s}^{-1}$ half peak-to-peak. We see that method (B) with the inversion shows rather a sinusoidal magnetic field oscillation response (thin solid) as compared to method (A) which looks more like triangular oscillations (dotted). Furthermore, the mean magnetic field of method (B) stays at $2000 \mathrm{G}$ and shows a peak-to-peak value of $\approx 26 \mathrm{G}$. The phase of shift of 90 degrees for the magnetic field in comparison to the

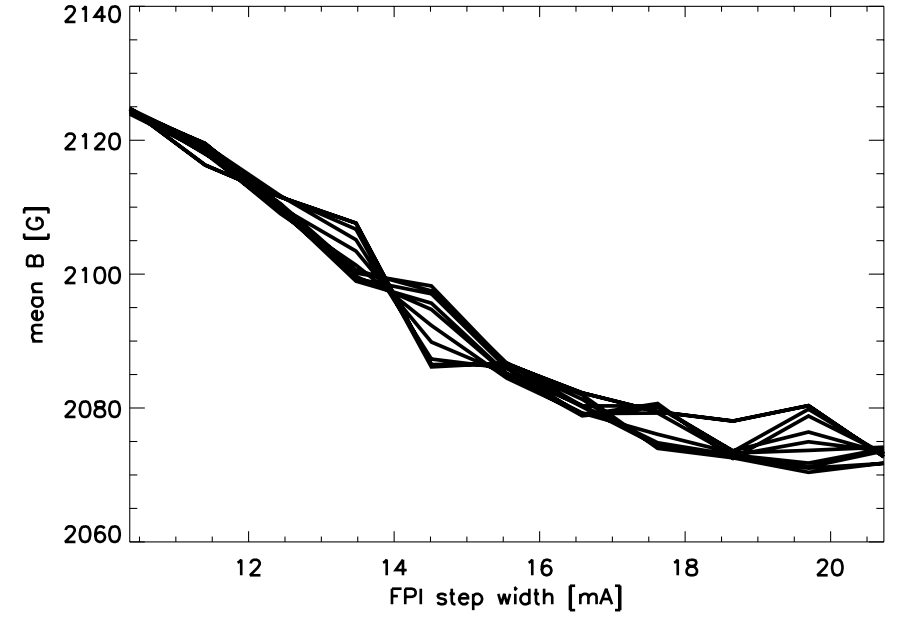

Fig. 5. Mean magnetic field for a 3 min velocity oscillation, several velocities and step-widths of the FPI spectrometer, calculated with method (A). With the inversion method (B) one would measure $2000 \mathrm{G}$ constantly.

velocities can be explained as follows. When the velocity change is small, as it is the case when we are near the maximum of a sinusoidal curve, the whole profile is shifted without strong deformation. Thus the the magnetic field strength measured is nearly undisturbed. Instead, if the velocity is close to zero and is changing its sign, the velocity change during the scanning process is very large and the line profile will be strongly deformed: an extreme value of the erroneous magnetic field occurs. Furthermore, if we start at negative velocities (down-flow) and change to positive velocities (up-flow) during the scan, the first lobe is red-shifted, while the second lobe is blue-shifted in the spectrum and we measure a minimum in the derived magnetic field. Consequently, when we start from positive velocities, we will find a maximum value. Of course, this will reverse if we change the scan direction and, the magnetic field will the lead the velocities by 90 degrees. This effect can not only be produced by observed oscillations. If a single shock wave is running through the area observed, the velocity gradient in time will be large causing a strong but nevertheless artificial magnetic field change.

We also compared the influence of different velocity wave amplitudes with method (A). For Fig. 3 we also used a FPI step size of $16.59 \mathrm{m \AA}$. Not surprisingly, a higher driving velocity amplitude leads to a larger error in the magnetic field oscillation. Again, there should be no oscillation in the magnetic field at all. The shorter the period of the oscillation, the stronger the effect of line profile deformation, as the velocity changes faster during the scan process.

In Fig. 4 we demonstrate the dependence of the erroneous magnetic field oscillation on the frequency. The velocity amplitude used is $250 \mathrm{~m} \mathrm{~s}^{-1}$, the periods $(P)$ of the oscillations are given relatively to the measurement time of $T=25.5 \mathrm{~s}$. Of course, the magnetic field oscillation is zero at $P / T=1$. The erroneous amplitudes do not to converge to zero in the range of interesting periods. If the period of oscillation is smaller than the scanning time, 
the measurement will not reproduce the real periods but beat periods instead. These periods will influence the result strongly, as can be seen by the increasing magnetic field amplitude.

A further test shows the dependence of the mean magnetic field on the step size of the spectrometer for method (A), while all other parameters are kept the same as described in Sect. 2.1. We focus on a 3 min oscillation, and show calculations of different input velocities and derive the magnetic field with method (A). Looking at Fig. 5 one can see that the mean magnetic field decreases, if we increase the distance in wavelength between steps. The undisturbed and prescribed profile shows a much larger magnetic field $(2161 \mathrm{G})$. The precision of the profile obtained also changes the precision of the magnetic field strength. If we measure fewer profile points in the simulation, the error increases. One should keep in mind however that this is valid for method (A) only, method (B) always reproduced a mean value of $2000 \mathrm{G}$.

Without a confirming calculation for this instrument one should also mention that stronger magnetic fields yield larger errors, while a smaller field is not so sensitive (see MDI simulation). The two lobes are more strongly separated for large fields and therefore the time to scan the two lobes increases as does the error.

\section{MDI-simulation}

\subsection{Simulation}

We used artificial Stokes $(I+V)$ and $(I-V)$ profiles of Ni $676.778 \mathrm{~nm}$ calculated by DIAMAG with the same model atmosphere as before pervaded by a set of magnetic field strengths from $800 \mathrm{G}$ to $2400 \mathrm{G}$ in steps of $400 \mathrm{G}$.

We assumed local thermodynamic equilibrium (LTE). This assumption seems to be sufficient because non-LTE considerations introduce rather small effects on the line source function and the line opacity (Bruls 1993) and since we are interested in the relative variation of the line profiles caused by the process of measurement and the variation of the underlying velocity field no other thermodynamic parameter is involved. In the line profile computation we have accounted for radiative, van der Waals and for Stark broadening. The non-thermal broadening of micro- and macroturbulence were set to $500 \mathrm{~m} \mathrm{~s}^{-1}$. The assumption of that relatively small non-thermal (symmetric) broadening do not have any effect on the results of our simulations. We have calculated the profiles for a pure longitudinal magnetic field (inclination of $0^{\circ}$ to the line of sight) since the magnetic field strength determination of MDI provides an estimation of the longitudinal component only and we are not interested in spurious field strength introduced by a variation of the field inclination.

The profiles were shifted by a sine wave with different amplitudes and periods. The measuring procedure of MDI (see Scherrer et al. 1995) was simulated as follows. MDI is using a cadence of filters separated by $3 \mathrm{~s}$ : L1, R1, R2, L2, FC, L3, R3, R4, L4, FC, where L denotes left circularly polarized light and $\mathrm{R}$ right circular polarized light. FC is are continuum filtergrams which are not used in the simulation. The total sequence needs $30 \mathrm{~s}$. The chronological order of the filtergrams is important for the results, changing the order will change the results! The transmission of the filters (a combination of a Lyot filter and the Michelson-interferometer) were multiplied to the appropriate Stokes profiles, then the total intensity was calculated by integration. These intensity values were combined for each polarization to get an $\alpha_{\mathrm{L} / \mathrm{R}}$-value which is used to derive the velocity $\left(V_{\mathrm{L}}, V_{\mathrm{R}}\right)$ with the help of a lookup table (see Scherrer et al. 1995), this is the center of gravity method. The magnetogram value is then $B=\left(V_{\mathrm{R}}-V_{\mathrm{L}}\right) / 2.84$ and the velocity in circular polarized light is $V=0.5 \cdot\left(V_{\mathrm{R}}+V_{\mathrm{L}}\right)$. We calculated $100 \mathrm{MDI}$ scans for each $V, B$-input pair and calculated the rms values of the measured $V$ and $B$.

The difference of this simulation to the ones described in Ruedi et al. $(1998,1999)$ is that we take into account the change of the line profile during the scanning sequence because of the velocity oscillation while Ruedi et al. just used an unchanging doppler-shifted profile which is less realistic and leads to a $B$ oscillation which is in antiphase with the oscillation of the velocity (compare Figs. 2 and 5 in Ruedi et al. 1998). Using an offset of zero in the velocity oscillation and having in mind the symmetry of the lookup table the method of Ruedi et al. will show a $B$ oscillation with twice the frequency of the velocity (not shown in the mentioned paper).

\subsection{Results and discussion}

The general remarks are the same for MDI and FPI, for a detailed description of the effect see Sect. 2.3.

The simulated oscillations look similar to Fig. 2 in the FPI case, only the phase shift is different. The phase shift in the simulation between $(\delta V, \delta B)$ is -90 degrees like found by Norton et al. (1999) in measurements. But the amplitudes in the measurements are much larger than predicted by our simulation so it seems to be of different origin. Reversing the scanning sequence of the filters will change this phase to be 90 degrees like in the FPI spectrometer case.

Prescribed sinusoidal velocities with amplitudes of $10 \mathrm{~m} \mathrm{~s}^{-1}$ were not reproduced by the simulation, the output values in these cases were zero. The values shown in the Fig. 6 are always values of the output of the simulation and not input values therefore they should be comparable to observations. Magnetic field strengths were overestimated by the simulation compared to the input values in the spectral line formation code by up to $8 \%$. This is similar to the FPI spectrometer case without using inversion.

The apparent oscillation in the magnetic field strength for several rms velocities and mean magnetic field strengths are plotted in Fig. 6. The error is a function of the underlying velocity oscillation in amplitude, frequency and mean value of velocity and also of mean value of the 

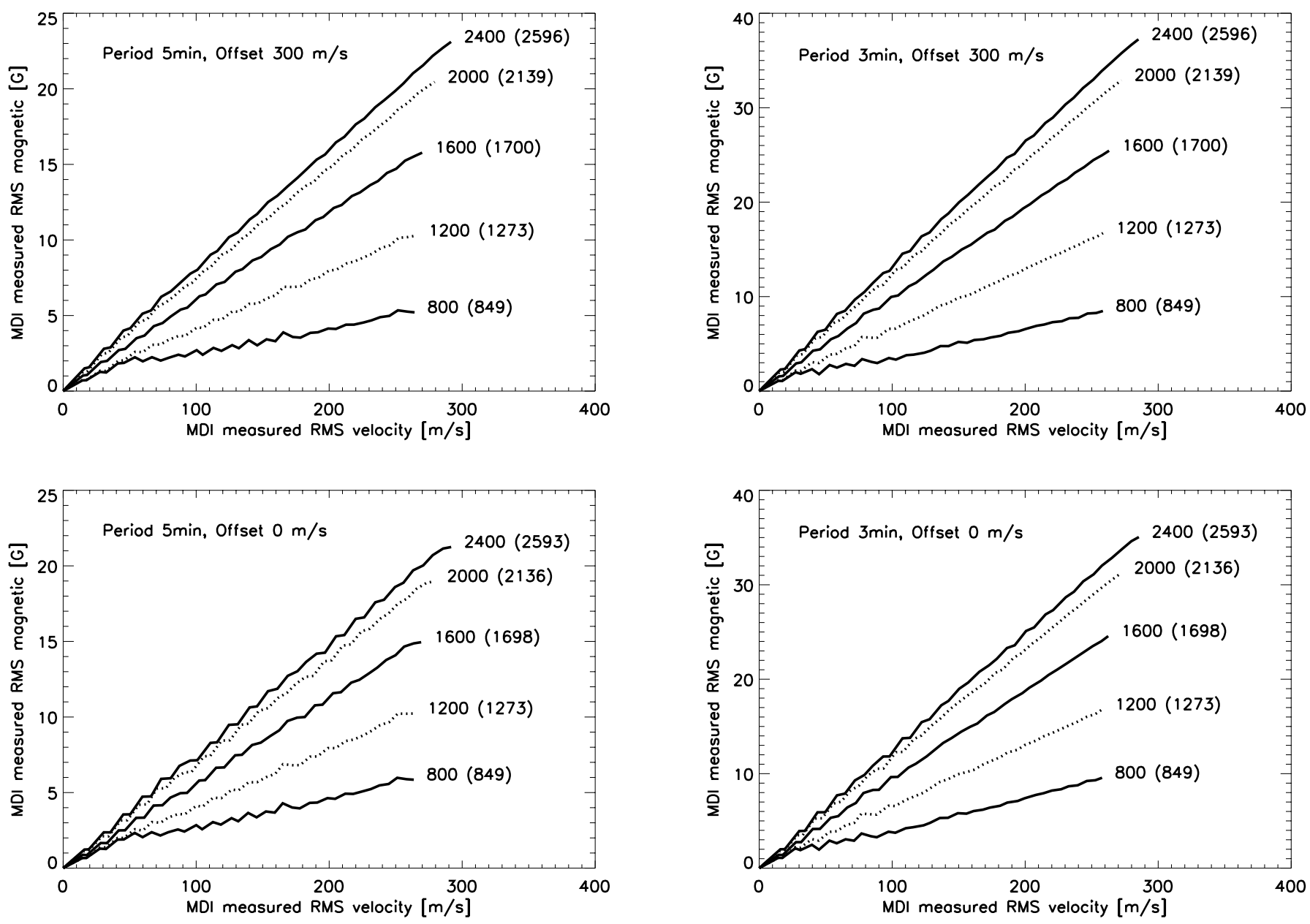

Fig. 6. The rms values of the apparent magnetic field oscillation are plotted versus the rms of the 3 min and 5 min velocity oscillation. The prescribed magnetic field in Gauss is given while the mean magnetic field derived from the MDI simulation is written in brackets. There are plots with and without offset in the prescribed velocity.

magnetic field. We show calculations for $3 \mathrm{~min}$ and $5 \mathrm{~min}$. The rms values of the erroneous magnetic field increase with increasing velocities amplitudes and mean magnetic fields. The offset in the velocity (due to solar rotation) increases the error for larger magnetic fields and decreases the error for smaller magnetic fields. Shorter periods of oscillations lead to larger errors (see FPI for comparison). For a rms in velocity of about $100 \mathrm{~m} \mathrm{~s}^{-1}$ we get magnetic field rms amplitudes of $7 \mathrm{G}$ for the $5 \mathrm{~min}$ case and $12 \mathrm{G}$ for the $3 \mathrm{~min}$ case for a magnetic field strength of $2000 \mathrm{G}$ and an offset in velocity of $300 \mathrm{~ms}^{-1}$. Comparing to the mean magnetic field this is neglectable $(0.3 \%)$, but when looking at oscillations it might increase up to $25 \%$ of the rms of the time series, which means it should not be neglectable any more. The power spectrum of velocity and magnetic field of the leading spot in Fig. 3 of Ruedi et al. (1998) shows a peak which is in the range of these errors. It might be an implication of the method where the mean of the time series of the sunspot where analysed and not a mean of the power spectra were taken.

\section{Conclusions}

We have found a cross-talk from a simple change of velocity to an apparent change in the magnetic field strength caused only by the measuring method of sequentially scanning through a spectral line profile in time. This is especially of interest when small oscillations in the magnetic field strength are to be observed or when shock waves are analysed that result in large velocity gradients. The effect is very small: a change of the separation of the Stokes $V$ lobes of about $0.7 \%$ in FeI $617.3 \mathrm{~nm}$ can lead to a magnetic field strength change of $15 \mathrm{G}$.

The simulated cross-talk in the case of the FPI spectrometer is of the same order of magnitude as the observed magnetic field oscillations of Horn et al. (1997) and Balthasar (1999). However, one has to be careful in interpretation because the phase shift seems to be different. Horn et al. (1997) found a zero phase shift between the velocity and the magnetic field which cannot be explained by this cross-talk, because we expect it to be 90 degrees. Also, when comparing our simulation to actual measurements, we would expect to find a larger number of reports 
of (erroneous) magnetic field oscillations as velocity oscillations in active solar regions can frequently be found. It might be that seeing effects disturb the line profile much more than this systematic effect.

The simulated cross-talk in the case of MDI on board SOHO is of the same origin and magnitude as the one of the FPI spectrometer. The expected phase shift between velocity and magnetic field oscillations introduced by the cross-talk is -90 degrees for MDI. The actual phases observed in MDI data have a wide range of values, depending in part upon type of magnetic feature and center-to-limb angle (Norton et al. 2001). Furthermore, MDI data shows velocity and magnetic oscillations which are not co-spatial. These facts combined with the knowledge that the observed magnetic oscillations have higher amplitudes than those predicted by this simulation indicate that the temporal behaviour of MDI magnetic data is not dominated by this cross-talk mechanism. However, there may be portions of the solar disk where no true magnetic variations exists and where the phase values are determined by this mechanism.

As a consequence of our simulation we would encourage observers to simulate the behaviour of the used setup accurately and to take into account the cross-talk as described herein before interpreting magnetic field data. Furthermore we suggest not to use simple magnetic field analysis e.g. by fitting the lobes but to use a full inversion instead with low resolution wavelength data as produced by a FPI spectrometer. Also, when changing the experimental setup, preference should be given to the FPI recording speed.

Velocity measurements as derived from a FPI spectrometer or MDI were not strongly affected. So it should be possible to reduce or even remove the error of this systematic effect using the measured velocities and mean magnetic fields. We have the following suggestion which is not straightforward and will be examined in future. If one is looking for oscillations and using power spectra, one can use the information of the power spectrum (frequency, amplitude and phase) of the velocity oscillation to produce a full continuous time series. With this continuous time series one can simulate the measuring process of the instrument as described in Sects. 2.1 and 3.1. Then there are two possibilities: if the original velocity time series is perfectly reproduced by this simulation, one should subtract the artificial magnetic field time series from the one of the measured data. If it is not perfectly reproduced but the power spectra is still correct one can subtract the power spectra of the of the artificial magnetic field oscillation from the measured one.

Acknowledgements. We thank J. Staude for reading the manuscript and helpful suggestions. Furthermore we would like to thank the referees for their critical and constructive comments and suggestions which have improved the quality of the paper.

\section{References}

Balthasar, H. 1999, Solar Phys., 187, 389

Bendlin, C., Volkmer, R., \& Kneer, F. 1992, A\&A, 257, 817

Bendlin, C., \& Volkmer, R. 1993, A\&A, 278, 601

Bendlin, C., \& Volkmer, R. 1995, A\&AS, 112, 371

Bruls, J. H. M. J. 1993, A\&A, 269, 509

Grossmann-Doerth, U. 1994, A\&A, 285, 1012

Horn, T., Hofmann, A., \& Balthasar, H. 1996, Solar Phys., 164, 321

Horn, T., Staude, J., \& Landgraf, V. 1997, Solar Phys., 172, 69

Kollatschny, W., Wiehr, E., Stellmacher, G., \& Falipou, M. A. 1980, A\&A, 86, 245

Norton, A. A, Ulrich, R. K., Bush, R. I., \& Tarbell, T. D. 1999, ApJ, 518, L123

Norton, A. A., Ulrich, R. K., \& Liu, Y. 2001, ApJ, 561, 435

Rüedi, I., Solanki, S. K., Stenflo, J. O., Tarbell, T., \& Scherrer, P. H. 1998, A\&A, 335, L97

Rüedi, I., Solanki, S. K., Bogdan, T., \& Cally, P. 1999, Proc. of the 2nd Solar Polarisation Workshop, Solar Polarisation, Astrophys. Space Sci. Library, 243, 337

Scherrer, P. H., and the MDI Team, 1995, Solar Phys., 162, 129 